\title{
MERCADOS INFORMALES, INTERCAMBIOS Y CESIONES DE AGUA DE RIEGO EN LA COMUNIDAD VALENCIANA
}

\author{
García-Mollá, M. ${ }^{1}$, Sanchis-Ibor, $C^{2}{ }^{2}$, Torregrosa Martí, $T^{3}$, Sevilla Jiménez, $M{ }^{4}$
}

\author{
${ }^{1}$ Profesora titular, Centro Valenciano de Estudios del Riego, Universitat Politècnica de \\ València, mgarmo@esp.upv.es \\ ${ }^{2}$ Investigador, Centro Valenciano de Estudios del Riego, Universitat Politècnica de València, \\ csanchis@hma.upv.es \\ ${ }^{3}$ Profesora titular, Departamento de Análisis Económico Aplicado, Universitat d'Alacant, \\ teresa.torregrosa@ua.es \\ ${ }^{4}$ Catedrático, Departamento de Análisis Económico Aplicado, Universitat d'Alacant, \\ martin.sevilla@ua.es.
}

\section{Resumen}

A inicios de la pasada década la legislación española de aguas incorporó diversos cambios normativos para permitir la realización de cesiones e intercambios de recursos hídricos entre concesionarios y usuarios, con el ánimo de flexibilizar la asignación de recursos y mejorar su aprovechamiento, particularmente en contextos de escasez. Sin embargo, como ya han apuntado diversos autores, con anterioridad a estos cambios legales ya existían fórmulas de cesión e intercambio de recursos entre usuarios, más o menos formales. El objetivo del presente trabajo es analizar las cesiones e intercambios de aguas que han tenido lugar en la Comunidad Valenciana en las últimas décadas. Los resultados demuestran que los intercambios de derechos entre usuarios pueden resultar eficaces para resolver problemas locales de escasez. El trabajo discute también el concepto de mercados informales y si estos intercambios o cesiones pueden considerarse como tales.

\section{1- Introducción}

La asignación del agua en España ha sido tradicionalmente realizada bajo los preceptos del modelo de oferta (Carles Genovés, 2001). En este modelo el objetivo fundamental era llevar al agua al usuario con un coste bajo para fomentar el despegue económico. En coherencia con este modelo, la asignación de recursos se realizaba -y se sigue realizando- mediante el sistema concesional. En este sistema las concesiones son generosas y los precios son bajos para fomentar el crecimiento económico. Por el contrario, es un sistema poco flexible y poco eficiente económicamente.

El crecimiento de las demandas de agua para riego y para otros usos ha hecho que en las cuencas del sur y del este mediterráneo se llegue al "cierre de cuenca" (Palomo-Hierro \& Gómez-Limón, 2014). Según Molle et al., (2010) esta situación de cierre de cuenca se alcanza cuando los compromisos adquiridos con los usuarios no se cumplen durante todo el año. En este contexto no se pueden aumentar demandas sin disminuir otras ya existentes (Falkenmark \& Molden, 2008) y continuar con el modelo de oferta únicamente incrementaría la presión sobre los recursos existentes (Molle et al., 2010).

En estas circunstancias deben buscarse nuevos instrumentos de asignación del recurso que permitan la flexibilización de las concesiones y que faciliten una asignación más eficiente del 
recurso. Los mercados de agua son un instrumento, relativamente reciente, que trata de mejorar la eficiencia sin que disminuyan los beneficios de los derechos de los poseedores de los derechos de propiedad. Dinar et al. (1998) enumeran las ventajas e inconvenientes del mercado como mecanismo de asignación del agua. Entre las ventajas de este mecanismo está el aumento de beneficios que puede experimentar el comprador y vendedor. En caso de que las transacciones sean del ámbito agrario al urbano puede disminuir la contaminación relacionada con el exceso de riego y puede inducir a mejorar la tecnología de riego, ya que informa a los usuarios sobre el coste de oportunidad. Entre las desventajas que estos autores encuentran están las dificultades para medir el agua, definir los derechos de propiedad, identificar y compensar a los terceros afectados y las externalidades, así como las posibles degradaciones ambientales. Otro problema surge de la dificultad de establecer mercados globales limitándose las posibilidades a mercados locales.

La aprobación de la de la Ley 46/1999 estaba encaminada a dotar de mayor flexibilidad al sistema permitiendo a los poseedores de concesiones venderlas temporalmente a otros concesionarios de mayor o igual rango al precio que acuerden mutuamente. Además la Ley prevé la creación de los bancos de agua o centros de intercambio. Los centros de intercambio pueden funcionar únicamente en situaciones excepcionales en las que los organismos de cuenca pueden realizar una oferta pública de adquisición de derechos a un precio determinado para, después, realizar una oferta pública de venta de todos o parte de los volúmenes adquiridos. Desde su aprobación la actividad de estos mercados ha sido escasa (Palomo-Hierro, Gómez-Limón \& Riesgo, 2015) y, aunque tanto el número como los volúmenes intercambiados aumentaron durante la sequía 2005-2008, los volúmenes intercambiados no llegaron al $1 \%$ de los consumidos en las zonas receptoras (Garrido, Rey, \& Calatrava, 2012). Según Hernández-Mora y De Stefano (2013) esto puede deberse a las reticencias de los agricultores con derechos tradicionales de uso de agua, ya que estarían poniendo de manifiesto que las concesiones existentes exceden a sus necesidades reales.

Pero tradicionalmente los agricultores han buscado métodos de flexibilizar el rígido sistema concesional existente, como las subastas de agua o cesiones de agua entre usuarios de una misma comunidad de regantes o entidades cercanas en momentos de sequía (Torregrosa 2009; García Mollá, 2000). Algunos de estos intercambios han sido definidos como mercados informales. Según Hernández-Mora y De Stefano (2013) los mercados informales son aquellas operaciones de compra-venta de agua que se producen al margen de los supuestos que contempla la legislación de aguas vigente y en ocasiones sin control por parte de la administración competente. Afectan tanto a derechos privados de aguas subterráneas como a aguas sujetas a concesión u otros títulos de aprovechamiento. Estas transacciones se producen en aquellas zonas donde las administraciones públicas no han podido dar respuesta a los problemas locales de escasez.

Los mercados informales han sido empleados en muchos países como Australia, India, Pakistán o Méjico (Brozovic, Care, \& Sunding, 2002; Meinzen-Dick, 1998; Rosegrant \& Binswanger, 1994; Saleth, 1998). Habitualmente se llevan a cabo mediante simples acuerdos entre regantes en los que la administración prácticamente no interviene y con unos costes de transacción muy pequeños. Los mercados formales, sin embargo, han sido menos implementados ya que requieren que los derechos al uso del agua estén claramente definidos y registrados y su uso controlado y medido (Bjornlund, Nicol, \& Klein, 2009). Esto implica que se debe llegar a acuerdo más complejos, con costes de transacción más elevados y con una intervención directa de las administraciones públicas que es cuanto menos complicada en los países en desarrollo (Bjornlund, 2004). 
El objetivo de esta comunicación es estudiar cuatro casos de acuerdos de cesión o compraventa de aguas entre usuarios agrarios o entre usuarios agrarios y urbanos en la Comunidad Valenciana en las últimas décadas. Buena parte de estas transacciones se han producido sin que sea necesaria la intervención de la administración, a veces buscando fórmulas legales diferentes a los supuestos contemplados para el intercambio de recursos por la Ley de Aguas y otras simplemente al margen de este ordenamiento.

\section{2- Las cesiones de agua entre usuarios agrarios.}

\section{1- Las cesiones de aguas superficiales sobrantes en entidades de riegos tradicionales.}

Los procesos de expansión histórica de los regadíos valencianos de aguas superficiales se desarrollaron, desde época islámica y hasta el siglo XIX, sobre espacios en los que ya existían pequeñas áreas regables dispersas abastecidas por aguas subterráneas. En la mayoría de los casos, estos sistemas de riego se conectaron e integraron en las redes de agua superficial. Sin embargo, en los humedales costeros del golfo de Valencia, estos microsistemas de riego mantuvieron su independencia institucional, aunque desarrollaron una dependencia creciente de los caudales sobrantes o "caigudes" del riego superficial. Hoy día subsisten entidades de riego de este tipo vinculadas a tres regadíos de aguas superficiales, los de la Acequia Real del Júcar (ARJ), las acequias de la Vega de Valencia y la Real Acequia de Moncada (RAM).

En el área regable de la ARJ subsisten dos entidades de riego independientes, la Acequia de la Huerta de Sollana y el Sindicato de las Partidas Arroceras de Albalat de la Ribera. En ambos casos se trata de entidades de riego con pequeñas concesiones de aguas subterráneas en surgencias naturales, cuyos caudales han mermado notablemente por la explotación del acuífero de la Plana de Valencia mediante pozos. A efectos prácticos ambas se abastecen de forma permanente casi exclusivamente de los caudales que les proporciona la ARJ, el riego se coordina entre ambas instituciones sin que exista ningún compromiso escrito para la cesión de recursos. La transformación del área regable de la ARJ a riego localizado, actualmente en marcha, va a suponer un cambio en estos procedimientos. En el caso de Albalat de la Ribera, las tierras de huerta y cítricos van a proceder a la implantación del riego localizado y a integrarse a todos los efectos en la ARJ, mientras que el arrozal mantendrá su independencia y el procedimiento tradicional de cesión de recursos. La Acequia de la Huerta de Sollana no ha sido incluida en los planes de modernización de la ARJ y no está prevista su integración en esta institución, por lo que queda pendiente para el futuro como se resolverá su abastecimiento una vez que los sectores contiguos de la ARJ adopten el riego presurizado.

En la Huerta de Valencia, existen dos tipologías de cesiones. Por un lado, comunidades de regantes constituidas como el Sindicato de Riego de la Marjal de Massanassa, que dispone de concesiones administrativas de determinadas fuentes de la marjal de l'Albufera, pero que depende casi en absoluto de los aportes de la Acequia de Favara, derivada del Turia. Este canal también abastece otras dos áreas regables, las cuales carecen de presencia institucional y de concesión administrativa de aguas, se trata de las tierras de la marjal de Sedaví y de la jurisdicción de Francos, Marjales y Extremales de la ciudad de Valencia, también abastecida por la Acequia de Rovella. Estas dos últimas constituyen un caso inusual en el ordenamiento jurídico del riego español, por cuanto la gestión de la zona regable la desarrolla la administración municipal y se carece de concesión administrativa de aguas. Se trata por tanto, de aprovechamientos que mantienen la situación jurídica previa a la Ley de Aguas de 1866 y que no disponen de garantías legales de suministro. 
El caso de la Comunidad de Regantes de las Tierras Arrozales de El Puig, enclavada en la RAM, presenta una génesis más compleja y se halla en una situación más conflictiva. Las tierras de la RAM se dividían históricamente en dos sectores, el "jovedat" con derechos plenos de usos de aguas, y el "extremal" con derecho a uso de aguas sobrantes (Sales, 1986). Las tierras dedicadas al arroz en el extremal de El Puig, debido a la necesidad de mecanismos específicos de gestión del riego, constituyeron en 1890 la Comunidad de Regantes de las Tierras Arrozales de El Puig a partir de unas escuetas concesiones de aguas subterráneas. Para proceder a la inundación primaveral del arrozal, se estableció un procedimiento denominado la "retancà" que permitía cerrar durante dos días todas las tomas de la RAM para ceder el agua a estas partidas de marjal. El pasado siglo, al regularse el río Turia con el embalse de Benagéber, se incrementó la garantía de suministro y se pudo proceder a la completa integración del extremal en la RAM mediante un proceso que ha estudiado Sales (in fieri). La integración del extremal no implicó ni la supresión de la retancà, ni la desaparición de la comunidad de regantes del arrozal. Como consecuencia de ello, estas tierras están hoy día adscritas a dos comunidades de regantes y reciben recursos de ambas, situación que queda fuera del ordenamiento jurídico de aguas.

\section{2- Las ventas de aguas residuales entre comunidades de regantes.}

A inicios de la década de 1980, ante la necesidad de dar salida a los caudales que iban a ser tratados por la estación depuradora, un grupo de propietarios agrarios del municipio negoció con el Ayuntamiento de Torrevieja la cesión de dichas aguas para su uso agrícola. Construyeron una estación de bombeo y una red de canales para su distribución, repartiendo los costos entre los interesados. A éstos se les añadieron algunos pequeños propietarios cuyas tierras resultaron afectadas por el trazado del sistema de riego, los cuales se interesaron en aprovechar estos recursos para el riego. En 1982, los regantes comenzaron a recibir aguas residuales depuradas, pero no fue hasta la década siguiente, en 1994, cuando constituyeron la Comunidad de Regantes de Torremiguel, a fin de legalizar la este aprovechamiento y conseguir la concesión administrativa de caudales.

La comunidad de regantes disponía inicialmente de una concesión de las aguas residuales depuradas, otorgada por el Ayuntamiento de Torrevieja, por un volumen de $2.140 .000 \mathrm{~m}^{3}$. Sin embargo, el acelerado desarrollo urbano de Torrevieja incrementó considerablemente el efluente de la estación depuradora, que hubo de ser ampliada en dos ocasiones, en 1990 y 1994. En consecuencia, los caudales administrados por la comunidad de regantes también fueron aumentando, haciendo necesaria la actualización de la concesión administrativa. Así en 2003, la concesión otorgada por la Confederación Hidrográfica del Segura se elevó hasta los $5.446 .000 \mathrm{~m}^{3}$ anuales, a razón de unos $6.800 \mathrm{~m}^{3} / \mathrm{ha} / a n ̃ o$. Sin embargo, el ritmo de crecimiento de Torrevieja, que entre 1982 y 2012 pasó de 12.321 a 103.720 habitantes, hizo que con anterioridad a la actualización de la concesión, ya se superara con creces el volumen del efluente reutilizado. En el año 2002, con 50.953 habitantes, ya se habían absorbido más de $6.000 .000 \mathrm{~m}^{3}$ y se esperaba un incremento continuado de los recursos, en paralelo al crecimiento urbano de dicho núcleo turístico-residencial.

La concesión de aguas de la EDAR de Torrevieja eximía a los regantes de efectuar pago alguno por los caudales recibidos, de modo la comunidad podía distribuir agua a muy bajo coste a sus usuarios. Sin embargo, a su vez, la comunidad de regantes se comprometía a utilizar la totalidad de los caudales, y por tanto evitar su vertido a la línea de costa, a fin de garantizar la calidad de las aguas de las playas. Debido al desorbitado crecimiento de Torrevieja, el cumplimiento de esta condición comenzó a resultar complicado, porque el efluente estaba superando la capacidad de absorción de las fincas agrícolas y los cuatro campos de golf abastecidos por la comunidad, y no se disponía de autorización de vertido. 
Por ello entre los años 2000 y 2007, la comunidad optó por vender agua a las comunidades de regantes vecinas de Campo de Salinas y San Miguel de Salinas, pertenecientes a la zona regable de La Pedrera, vinculada al Trasvase Tajo-Segura. Estas ventas se produjeron en un contexto de enorme escasez en la cuenca del Segura. Inmediatamente, otras comunidades de la zona regable se interesaron por estos recursos. En consecuencia, se construyó una red de canalizaciones para hacer posible la redistribución de los excedentes de la estación depuradora a otras entidades, como a la comunidad de regantes de Santo Domingo, también perteneciente a la Comunidad General de Usuarios de la Zona Regable de La Pedrera. Estas aguas se pagaban, mediada la pasada década a $0,10 € / \mathrm{m}^{3}$, precio que consideraba el valor de venta del agua y los costes de elevación.

Finalmente, en 2007, la Confederación Hidrográfica del Segura regularizó esta situación mediante una reordenación de los derechos concesionales de la EDAR de Torrevieja, otorgando una concesión administrativa de $1^{\prime} 77 \mathrm{hm}^{3}$ anuales a la Comunidad General de Usuarios de la Zona Regable de La Pedrera. De este modo, mediante la adscripción de la concesión a la comunidad general, se paralizaron las operaciones de venta.

\section{3- Las transacciones entre usuarios agrarios y urbanos}

\section{1- Cesiones de aguas subterráneas para abastecimiento urbano}

Los acuíferos de la cuenca del Vinalopó han sido, históricamente, una de las principales fuentes de aprovisionamiento urbano de la ciudad de Alicante y de otras áreas urbanas de su entorno metropolitano. El desarrollo de los regadíos de aguas subterráneas del Alto Vinalopó, singularmente durante en la segunda mitad del siglo XX, permitió la movilización de importantes volúmenes de recursos y facilitó el establecimiento de acuerdos entre las entidades de riego de la comarca y la empresa Aguas Municipalizadas de Alicante (AMA), con el fin de transferir recursos hacia los abastecimientos que atiende esta empresa en la comarca del Vinalopó Mitjà (Petrer, Novelda y Monforte del Cid) y en el área metropolitana de Alicante. Dos comunidades de regantes históricas de la comarca, las de la Vall de Beneixama y la Huerta y Partidas de Villena, facilitan hoy día el abastecimiento de poblaciones. Para la transferencia de estos recursos, los regantes y la empresa de abastecimientos optaron por la fórmula de la constitución de comunidades de usuarios.

La Comunidad de Regantes de la Vall de Beneixama, abrió en 1970 el pozo de Saleretes, en término de Camp de Mirra, constituyendo la comunidad de bienes Valle de Benejama junto a la empresa Aguas Municipalizadas de Alicante. La concesión administrativa permite la extracción de $2 \mathrm{hm}^{3}$ anuales, de los cuales 0 ' $5 \mathrm{hm}^{3}$ corresponden a la comunidad, que actualmente no los emplea, mientras que los restantes $1^{\prime} 5 \mathrm{hm}^{3}$ son utilizados por AMA. En este caso, dado que la comunidad de regantes no hace uso de su concesión, la gestión es ejecutada por la empresa de abastecimiento, por lo que no existen pagos o compensaciones por la explotación del pozo.

Por su parte, la comunidad de regantes de Huerta y Partidas de Villena es propietaria de 7 pozos, con unos derechos concesionales que ascienden a 14,5 hm $\mathrm{hm}^{3}$. De ellos, $7^{\prime} 2 \mathrm{hm}^{3}$ están asignados a una comunidad de usuarios que constituyen esta entidad de riegos y AMA desde el año 2003. En este caso, la comunidad de regantes es la que explota todos los pozos. Se ha reservado 3 '325 $\mathrm{hm}^{3}$ para uso agrícola y los restantes 3 ' $875 \mathrm{hm}^{3}$ están destinados a los abastecimientos urbanos. Las tarifas de aguas reflejan un mecanismo de

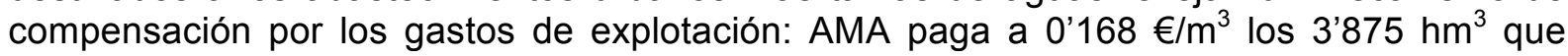
recibe anualmente, mientras que el costo del agua del mismo pozo para los socios de la comunidad de regantes es de $0^{\prime} 072 € / \mathrm{m}^{3}$. La diferencia en la asignación de los costes de obtención del recurso entre el abastecimiento y el riego refleja una proporción de 2,3 a 1 , 
cifra notablemente inferior a que la que suele aplicarse en el cálculo de los cánones y tarifas públicas.

\section{2- Las cesiones de aguas en el Consorcio de Aguas de la Marina Baixa}

La comarca de la Marina Baixa, al noreste de la provincia de Alicante, es uno de los nueve sistemas de explotación de la Confederación Hidrográfica del Júcar (CHJ). Ocupa $625 \mathrm{~km}^{2}$ y presenta una marcada dualidad geográfica, con una zona interior de relieves escarpados y alturas superiores a los $1.000 \mathrm{~m}$ en numerosas cotas, y unos piedemontes costeros masivamente ocupados por urbanizaciones turísticas de baja y alta densidad, como Altea, Benidorm o La Vila Joiosa.

La mayoría de los recursos hídricos son de procedencia subterránea, aproximadamente un $60 \%$, a los que hay que añadir un $30 \%$ de origen superficial y un $10 \%$ de aguas reutilizadas. Las fuentes de suministro son igualmente variadas. Los recursos subterráneos proceden las unidades hidrogeológicas de Aitana, Serrella-Aixortà-Algar y Orxeta; las aguas superficiales de los ríos Algar, Amadorio, Sella y Guadalest; y en cuanto a las aguas reutilizadas, proceden casi exclusivamente de tres EDAR, las de Benidorm, Altea y La Vila Joiosa.

En 1977, en un contexto de extrema sequía, la CHJ, la Diputación de Alicante y diversos municipios de la comarca crearon el Consorcio de Aguas de la Marina Baja (CAMB) con el objetivo de desarrollar, de forma coordinada, una adecuada gestión integrada de los recursos hídricos, a fin de satisfacer las demandas crecientes del consumo urbano sin desatender las necesidades agrícolas de agua.

El principal reto era poder cubrir adecuadamente los picos de demanda estacional principalmente veraniega-, generados por el núcleo de Benidorm y las áreas turísticas de su entorno. Durante estos periodos, los recursos concesionales asignados a los abastecimientos urbanos no eran suficientes para atender las demandas, por lo que era necesario movilizar nuevos recursos o hacer un uso más eficiente de los existentes. La forma de satisfacer estas necesidades puntuales era emplear el efluente de las depuradoras de las grandes zonas urbanas para el riego agrícola, con el fin de liberar parte de las aguas subterráneas para su uso en los abastecimientos urbanos.

En consecuencia, desde 1978 -y hasta nuestros días- el CAMB y las comunidades de regantes firman contratos privados de intercambio para proceder a la cesión a las zonas urbanas del agua subterránea asignada legalmente a los agricultores. No son contratos de cesión de derechos tal y como establece el artículo 67.1 del TRLA, ya que no existe esa cesión temporal de derechos, no estableciéndose además $\mathrm{m}^{3}$ intercambiados, precio de esos caudales, frecuencias, etc, sino que únicamente se establece, en ocasiones, el volumen máximo a intercambiar, la asignación económica que recibe la comunidad de regantes por parte del CAMB para el mantenimiento de infraestructuras y sufragar gastos relacionados.

Los volúmenes máximos varían anualmente en función de las disponibilidades de agua depurada, de las necesidades mensuales de agua para abastecimiento urbano, del régimen de lluvias, del mes del año, etc. En periodo estival son más elevados, ya que coinciden las bajas precipitaciones con los picos de la campaña turística y la de riegos. Las estimaciones de los volúmenes habitualmente objeto de intercambio, basadas en el consumo de aguas reutilizadas por parte de las comunidades de regantes, superan los $7 \mathrm{hm}^{3}$ anuales, teniendo el CAMB una capacidad de hasta $9 \mathrm{hm}^{3}$ para suministrar a los regantes. En algunas 
comunidades de regantes como la de Villajoyosa, los recursos depurados han llegado a suponer el $70 \%$ de las aguas consumidas (Torregrosa, 2009).

Estos intercambios también requieren de unas infraestructuras hidráulicas (estaciones de bombeo, embalses reguladores, conducciones de aguas blancas y conducciones de aguas depuradas) que han sido posibles gracias al soporte financiero del CAMB. Además, para mejorar la gestión integrada de recursos, el CAMB ha adquirido derechos de agua, que son destinados tanto a los abastecimientos municipales, como a los regantes que participan de los mecanismos de compensación de usos de las aguas.

\section{4- Discusión y conclusiones}

Las experiencias de cesiones e intercambios de aguas recogidas en este trabajo son una buena muestra de la capacidad de los regantes para alcanzar acuerdos que dan soluciones locales, permanentes o temporales, a determinados problemas de gestión del recurso, con independencia de la regulación que pueda establecerse en el marco jurídico del agua. También son un claro exponente de su aptitud para optimizar el aprovechamiento de los recursos hídricos. Los casos analizados merecen una consideración separada, por cuanto existen importantes diferencias, en su origen y en su naturaleza, entre las cesiones de aguas superficiales de los regadíos tradicionales del llano Júcar-Turia y los intercambios de aguas de las comarcas alicantinas.

Respecto a los regadíos tradicionales del Júcar y el Turia, nos hallamos ante una práctica plurisecular que ha encontrado diversas articulaciones institucionales, en casi todos los casos al margen de la legislación. Se trata de cesiones de agua sin compensación económica y que pese a su dilatada perduración en el tiempo, no han merecido su reconocimiento por la vía concesional.

La dependencia de muchos regantes respecto a estos recursos sobrantes les sitúa en una posición de debilidad, acentuada en aquellas zonas, como en los francos y marjales de Valencia y Sedaví, donde los usuarios no se han constituido en comunidad de regantes. De hecho, concesiones fluviales efectuadas con posterioridad a estos usos, anteriores y posteriores a la regulación fluvial, han permitido que otros usuarios les adelantaran en la prelación de usos, pese al principio prior in tempore, potior in iuris. Su integración en las comunidades de regantes que les subministran agua, como sucedió en algunos casos (acequias de Rascanya o Moncada), hubiera sido la opción más favorable a sus intereses. Las causas por las que algunas de estas entidades se integraron y otras no, merecen ser objeto de un estudio histórico e institucional más detallado (Sales, in fieri).

Pese a que las modestas dimensiones de estos riegos no suponen un problema relevante de gestión, la integración sigue siendo la opción más recomendable en la actualidad, singularmente en un contexto en el que la reducción de las superficies regadas debería estimular los procesos de revisión concesional. Además, como ha sucedido en el caso de Albalat de la Ribera (ARJ), las iniciativas de modernización del regadío deben servir para reducir la atomización de la gestión del riego y facilitar estos procesos de integración. Carece de sentido en todo caso la situación inusual, y de difícil justificación legal, de la comunidad de las Tierras Arrozales de El Puig, cuya área regable se solapa con la de la RAM. Una razón importante que aflora en este caso y que explica algunos de estos comportamientos, es la existencia de expectativas urbanísticas, que lleva a los responsables de algunas de estas pequeñas entidades a defender su independencia, a fin de obtener 
beneficios económicos en caso de expropiación de la superficie ocupada por la red de riegos y drenajes.

El caso de los intercambios y compra-venta de recursos de las comarcas alicantinas difiere sustancialmente de los anteriores. En todos estos ejemplos, el origen del intercambio de recursos se encuentra en el crecimiento de las áreas urbanas y al desarrollo del sector turístico. La irrupción de centenares de miles de nuevos usuarios urbanos ha generado una nueva necesidad -agua potable- y un nuevo recurso -aguas residuales-, redibujando el equilibrio entre oferta y demanda en varias comarcas alicantinas. Los intercambios identificados en estas tierras son en definitiva una adaptación de la gestión a un nuevo escenario hídrico, en la que las comunidades de regantes han desempeñado un papel clave, en tanto en cuanto ostentaban la titularidad legal de la mayor parte de los derechos de aguas.

En los ejemplos estudiados la rigidez que planteaba el régimen concesional se ha sorteado con diversos mecanismos para facilitar, con el concurso de los nuevos recursos -y el apoyo económico de la administración, en el caso del CAMB-, una gestión más eficaz. En el caso de Torrevieja se produce una respuesta de los usuarios a un problema coyuntural, que la administración ha reconducido por la vía de la asignación de nuevas concesiones. Los casos del Vinalopó y la Marina Baixa son acuerdos estables, de larga duración, en los que los usuarios han arbitrado compensaciones económicas a la explotación, cesión o sustitución de recursos. Son respuestas bien adaptadas a las condiciones y necesidades locales, que resultan satisfactorias para los usuarios y les otorgan agilidad y flexibilidad en la gestión, evitando controles y rigideces administrativas.

Pero los casos analizados, ¿Pueden considerarse mercados? Si seguimos los criterios aplicados por Hernández-Mora y De Stéfano (2012), podrían considerarse como tales. Sin embargo, por la escasa concurrencia de competidores y el limitado alcance de la oferta apenas si presentan las características que otros autores (Naredo, 2008) conceptualizan como mercado. Lo cierto es que si comparamos los casos aquí analizados con algunos de los que han sido expuestos (Meinzen-Dirk, 1988; Saleth, 1998; Bjornlund, 2004), aflora una significativa distancia en la oferta, la demanda y el volumen de transacciones. También el uso del término informales puede suscitar cierta controversia, por cuanto Bjornlund (2004) ha establecido la distinción entre mercados formales o informales en función del tipo de transferencia, permanente o temporal, de derechos de uso. Sin embargo, en España, se halla más extendido el uso de este término en función de si los intercambios siguen o no las fórmulas introducidas en la legislación de aguas en 1999. Todo esto probablemente hace necesario un análisis comparativo más amplio a fin de establecer una tipología más detallada de cesiones, intercambios y mercados.

Dejando de lado estos matices conceptuales, cabe hacer una valoración positiva de estas experiencias, que se han mostrado eficaces para resolver problemas locales. No obstante, sería recomendable, en estos y en otros casos de intercambios de recursos, un mayor control administrativo para evitar que se produjeran afecciones a terceros o impactos ambientales debido al cambio de uso del agua. La transparencia en la gestión de los recursos, que al fin y al cabo son públicos, es una cuenta pendiente de la administración del agua en España, y ésta atañe no solo a los organismos públicos, sino también a los aprovechamientos colectivos de titularidad privada.

\section{Bibliografía}

Bjornlund, H. (2004). Formal and informal water markets: Drivers of sustainable rural communities? Water Resources Research, 40, 1-12. doi:10.1029/2003WR002852 
Bjornlund, H., Nicol, L., \& Klein, K. K. (2009). The adoption of improved irrigation technology and management practices-A study of two irrigation districts in Alberta, Canada. Agricultural Water Management, 96, 121-131. doi:10.1016/j.agwat.2008.07.009

Brozovic, N., Care, J. M., \& Sunding, D. L. (2002). Trading activity in an informal agricultural water market: an example from California. Journal of Contemporary Water Research and Education, 121, 3-16.

Carles Genovés, J. (2001). La Administración Pública ante las nuevas políticas de aguas de la Directiva Marco. Il Congreso Ibérico Sobre Planificación Y Gestión de Aguas. Oporto 2001.

Dinar, A.; M. W. Rosegrant y R. Meizen-dick (1998) Water allocation mechanisms- principles and examples. World Bank. Agriculture and Natural Resources Departament

Falkenmark, M., \& Molden, D. (2008). Wake Up to Realities of River Basin Closure. International Journal of Water Resources Development, 24(2), 201-215. doi:10.1080/07900620701723570

García-Mollá, M. (2000). Análisis de la influencia de los costes en el consumo de agua en la agricultura valenciana. Caracterización de las entidades asociativas para riego, Tesis Doctoral, Universitat Politècnica de València.

Garrido, A., Rey, D., \& Calatrava, J. (2012). Water trading in Spain. Water, agriculture and the environment in Spain: can we square the circle, 205-216.

Hernández-Mora, N., \& De Stefano, L. (2013). Los mercados informales de aguas en España: una primera aproximación, 1-28.

Meinzen-Dick, R. S. (1998). Groundwater markets in Pakistan: Institutional development and productivity impacts. In Marketsfor Water (pp. 207-222). Springer US.

Molle, F., Wester, P., \& Hirsch, P. (2010). River basin closure: Processes, implications and responses. Agricultural Water Management, 97(4), 569-577. doi:10.1016/j.agwat.2009.01.004

Naredo, J. (2008). Lo público y lo privado, la planificación y el mercado, en la encrucijada actual de la gestión del agua en España. Panel Científico-Técnico de Seguimiento de La Política .... Retrieved from http://www.fnca.eu/phocadownload/P.CIENTIFICO/inf_mercado.pdf

Palomo-Hierro, S., \& Gómez-Limón, J. A. (2014). El papel de los mercados como instrumento para la reasignación del agua en España. Agua y Territorio, 1(2), 78-92.

Palomo-Hierro, S., Gómez-Limón, J., \& Riesgo, L. (2015). Water Markets in Spain: Performance and Challenges. Water, 7(2), 652-678. doi:10.3390/w7020652

Rosegrant, M., \& Binswanger, H. (1994). Markets in tradable water rights: potential for efficiency gains in developing country water resource allocation. World Development. Retrieved from http://www.sciencedirect.com/science/article/pii/0305750X94000751 
Torregrosa, T. (2009). La gestión del agua en la Marina Baja (Alicante) Temas de las Cortes Valencianas, $\mathrm{n}^{\circ} 19$.

Sales Martínez, V. (1988). La cuestión del extremal en el regadío de la Real Acequia deMoncada. Cuadernos de Geografía, 44, 221-234.

Sales Martínez, V. (in fieri). Las ampliaciones modernas en los regadíos históricos: el caso del extremal de la Acequia de Moncada, Tesis doctoral, Universitat Politècnica de València.

Saleth, R. (1998). Water markets in India: economic and institutional aspects. Markets for Water. Retrieved from http://link.springer.com/chapter/10.1007/978-0-585-32088-5_12 